\title{
Corrigendum: Mycobacterium
} tuberculosis Infection-Driven Foamy Macrophages and Their Implications in Tuberculosis Control as Targets for Host-Directed Therapy

\author{
Dahee Shim ${ }^{1,2}$, Hagyu Kim ${ }^{1}$ and Sung Jae Shin ${ }^{1 *}$ \\ ${ }^{1}$ Department of Microbiology, Institute for Immunology and Immunological Diseases, Brain Korea 21 Program for Leading \\ Universities and Students (PLUS) Project for Medical Science, Yonsei University College of Medicine, Seoul, South Korea, \\ ${ }^{2}$ Department of Life Science, Research Institute for Natural Sciences, College of Natural Sciences, Hanyang University, \\ Seoul, South Korea
}

Keywords: Mycobacterium tuberculosis, foamy macrophage, tuberculosis, immune responses, lipid metabolism, lung inflammation, host-directed therapy

\section{A Corrigendum on}

Mycobacterium tuberculosis Infection-Driven Foamy Macrophages and Their Implications in Tuberculosis Control as Targets for Host-Directed Therapy

by Shim, D., Kim, H., and Shin, S. J. (2020). Front. Immunol. 11:910. doi: 10.3389/fimmu.2020.00910

OPEN ACCESS

Approved by:

Frontiers Editorial Office,

Frontiers Media SA, Switzerland

*Correspondence:

Sung Jae Shin

sjshin@yuhs.ac

Specialty section:

This article was submitted to

Microbial Immunology,

a section of the journal

Frontiers in Immunology

Received: 08 June 2020 Accepted: 16 June 2020 Published: 17 July 2020

Citation:

Shim D, Kim H and Shin SJ (2020)

Corrigendum: Mycobacterium tuberculosis Infection-Driven Foamy Macrophages and Their Implications in Tuberculosis Control as Targets for Host-Directed Therapy.

Front. Immunol. 11:1601.

doi: 10.3389/fimmu.2020.01601
In the original article, incorrect information and references were included in Interaction Between Mtb and Mtb-Driven Foamy Macrophages, sub-section Characteristics of Mtb-infected Foamy Macrophages and the Utilization of Their Lipids by Mtb, Paragraph 3:

A reference in the sentence "Furthermore, it has been suggested the use of lipids in host cells is related to dormancy of Mtb." was incorrectly added as "Jaisinghani N, Dawa S, Singh K, Nandy A, Menon D, Bhandari PD, et al. Necrosis driven triglyceride synthesis primes macrophages for inflammation during Mycobacterium tuberculosis infection. Front Immunol. (2018) 9:1490. doi: 10.3389/fimmu.2018.01490." This should be deleted.

In addition, there was a further error within the same paragraph:

"The region of difference 1 protein in Mtb contributes to increasing the levels of intracellular triglycerides in Mtb by enhancing the expression of diacylglycerol $O$-acyltransferase, a key enzyme in triglyceride synthesis (68)."

The text should be substituted with the following:

"The region of difference 1 in pathogenic mycobacteria may contribute to the interaction between diacylglycerol $O$-acyltransferase, a key enzyme in triglyceride synthesis, and lipid droplets of mycobacteria to generate intracellular lipid inclusions." This statement is supported by the reference "Barisch C, Soldati T. Mycobacterium marinum degrades both triacylglycerols and phospholipids from its dictyostelium host to synthesise its own triacylglycerols and generate lipid inclusions. PLoS Pathog. (2017) 13:e1006095. doi: 10.1371/journal.ppat.1006095”.

The authors apologize for these errors and state that the revisions do not change the scientific conclusions of the article in any way. The original article has been updated. 


\section{REFERENCES}

68. Barisch C, Soldati T. Mycobacterium marinum degrades both triacylglycerols and phospholipids from its dictyostelium host to synthesise its own triacylglycerols and generate lipid inclusions. PLoS Pathog. (2017) 13:e1006095. doi: 10.1371/journal.ppat.1006095
Copyright $\odot 2020$ Shim, Kim and Shin. This is an open-access article distributed under the terms of the Creative Commons Attribution License (CC BY). The use, distribution or reproduction in other forums is permitted, provided the original author(s) and the copyright owner(s) are credited and that the original publication in this journal is cited, in accordance with accepted academic practice. No use, distribution or reproduction is permitted which does not comply with these terms. 